\title{
Treatment of the Myofascial Trigger-Points of Triceps Surae: A Systematic Review
}

\author{
Mathias Simon ${ }^{1 \#}$, Albert Pérez-Bellmunt ${ }^{1 *^{*}}$, Olivier Peillon ${ }^{1}$, Paolo Ragazzi $^{1}$, Alexandra \\ Myers Escolà ${ }^{1}$ and Carlos López-de-Celis ${ }^{2}$
}

${ }^{1}$ Department of Basic Areas, Universitat Internacional de Catalunya, Barcelona, Spain

${ }^{2}$ Department of Physical Therapy, Universitat Internacional de Catalunya, Barcelona, Spain

*Corresponding author: Albert Perez-Bellmunt, Universitat Internacional de Catalunya, c/Josep Trueta S/N, 08195 Sant Cugat del Vallès, Catalonia, Barcelona, Spain

\begin{abstract}
Background: Myofascial Trigger Points (MTrPs) in the triceps surae muscles are very frequent and their presence may be implicated in pain, such as nocturnal night cramps. These points cause important limitations to athletic performance and daily activities. For this reason, treatment of these MTrPs are essential in the clinical field and have been addressed by a large number of therapeutic strategies. Therefore, the aim of this review is to analyze treatments available for the MTrPs in the triceps surae and their effectiveness.
\end{abstract}

Methods: The PubMed and PEDro databases were both used for the study selection process. The terms "Trigger Points AND Gastrocnemius AND Soleus NOT Trapezius" were used and without a time restriction. From the 277 articles screened initially, 12 studies were selected to be included in this review. To assess the quality of the articles, the Jadad Scale and the U.S Preventive Task Force Scale were used.

Results: The studies reviewed included healthy subjects as well as patients with disorders, or pain affecting specifically the foot and the ankle. In these studies, both active and latent trigger-points were found. Of all the variables used, the treatment of the MTrPs allows for an improvement in jump performance, quality of life, insomnia, Range of Motion (ROM) of ankle dorsiflexion, mobility, and reduced pain. However, the MTrP therapy has no effects on depression or anxiety. The principal treatments used were injections, electrostimulation, dry needling, ischemic compression, and Extra-Corporeal Shock Wave Therapy (ESWT).

Conclusion: MTrPs can be the origin of several alterations. This review shows how the MTrPs treatment can improve those conditions.

\section{Introduction}

Myofascial Trigger Points (MTrPs) were initially defined as "a hyperirritable spot in skeletal muscle that is associated with a hypersensitive palpable nodule in a taut band" [1]. These irritable spots result painful with compression and can be related to a characteristic pain in predictable regional patterns within a referral zone (referring pain) [1,2]. According to the bibliography, the most frequent classification divides these MTrPs in Active Trigger Points (ATrPs) and in Latent Trigger Points (LTrPs). The etiologic theory of the MTrPs states that a motor end-plate dysfunction $[3,4]$ and a prolonged contraction of muscle fibers under the motor end-plate, are due to an excessive liberation of acetylcholine [5].

Although MTrPs are able to affect any muscle of the body or any of its parts, the triceps surae is described to be one of the most affected in lower limb. Their prevalence in this specific muscle is high $[6,7]$ and affects from $16 \%$ to $30 \%$ of healthy subjects [8]. In addition to their elevated incidence, the presence of MTrPs in gastrocnemius or soleus muscles is also associated with an alteration of muscle function, athletic performance, or an inability to carry out daily activities [9-12].

Although different techniques such as dry needling, stretching, digital is quemic pressure, and local injections of anesthetics are available for the treatment of MTrPs, to our knowledge no study has evaluated the different procedures used in the triceps surae. Therefore, the 
aim of this systematic review is to conduct a revision of scientific literature in order to identify which treatment is more effective, and the variables used to assess its effects on the subject's condition.

\section{Materials and Methods}

The bibliographic research was conducted using the following databases, MEDLINE and PEDro, inputting the following combination of words: "Trigger Points AND Gastrocnemius AND Soleus" and 418 articles were obtained. Based on title analysis of the first results, many articles were focused on the trapezius muscle. For this reason, the final search was narrowed down using the following combination of words: "Trigger Points AND Gastrocnemius AND Soleus NOT Trapezius". In the selection of articles, the year of publication was not taken into consideration.

Initially, 277 articles were eligible to be included in this revision. The articles were then narrowed down by the reviewers based on the study of the abstract, the title, the keywords and the inclusion criteria. Two independent reviewers made the first analysis, if a consensus was not reached, the opinion of a third reviewer was added. After the first analysis, 18 articles were selected. Finally, after their complete analysis using the full text, 12 of the selected were included in this revision and were studied in detail during in the assessment phase. Figure 1 represents the flowchart of the entire search process that was conducted until May $5^{\text {th }}, 2018$.

The INCLUSION CRITERIA of the present study were:

1. Trials involving gastrocnemius' MTrPs

2. Research that form part of the rehabilitation field.
3. Articles that can be obtained in full-text version and were published in one of the different languages spoken by the authors such as English, Spanish, Catalan, French, or Italian.

With the aim to assess the quality of the different articles included in this revision, the authors used the Jadad Scale, if possible, and the U.S. Preventive Task Force, both of which are standard in this type of investigation. The 3-item scale of Jadad, et al. [13] was used to assess the quality of the clinical trial. Scale scores can range from 0 to 5 points, with higher scores indicating a better quality (Table 1). The standard approach to evaluating the quality of individual studies was based on a hierarchical grading system of research design, in which randomized control trials received the highest score (Table 2) [14,15].

\section{Results}

\section{Level of evidence}

Out of the 277 articles found at the end of the first

Table 1: Preventive Services Task Force Hierarchy of research design U.S.

I Evidence obtained from at least one properly randomized controlled trial.

II-1 Evidence obtained from well-designed controlled trials without randomization.

II-2 Evidence obtained from well-designed cohort or case control analytic studies, preferably from more than one center or research group.

II-3 Evidence obtained from multiple time series with or without the intervention.

III Opinions of respected authorities, based on clinical experience, descriptive studies and case reports, or reports of expert committees.
Potentially relevant studies screened $(n=418)$

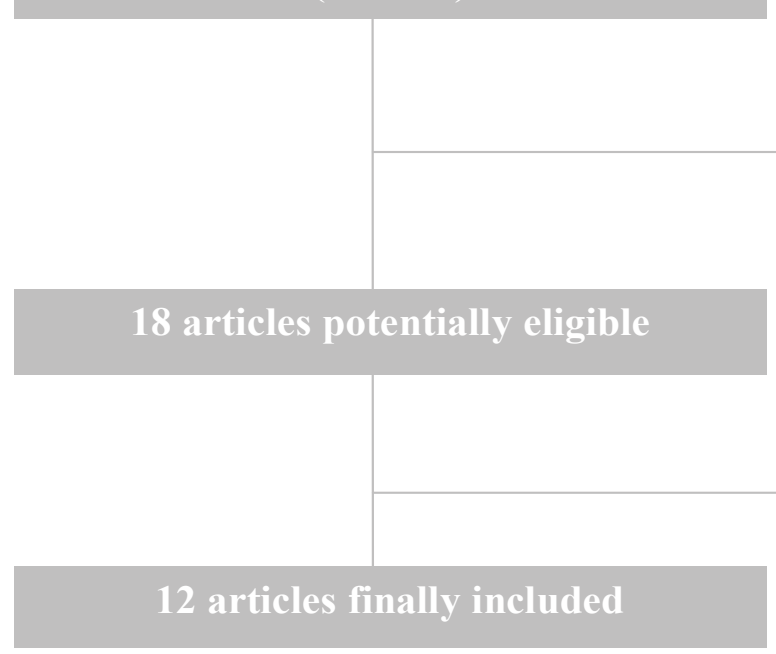

Excluded $(n=400)$

- Non-tricepssurae $(\mathrm{n}=343)$

- Non MTrPs $(\mathrm{n}=38)$

- Nonhuman studies $(\mathrm{n}=17)$

- Unknown language $(\mathrm{n}=1)$

- Case report $(\mathrm{n}=1)$

Excluded $(n=6)$

- Non-trials study $(\mathrm{n}=3)$

- Repeated study $(\mathrm{n}=1)$

- Non MTrPs study $(\mathrm{n}=2)$

Figure 1: Flowchart of the study selection. 
search, 12 articles were selected to use in this review of the scientific evidence (Table 3). Using the U.S. Preventative Task Force to evaluate the quality, it can be seen that most of the studies present a very high ( $66 \%$ of the studies) or medium level of evidence. Only two studies present a low level of evidence. Yet when evaluating the quality of the clinical trials with the Jadad scale, only two studies present a high-quality methodology (score higher than 4) while the rest of the articles present a low-quality methodology (score less than or equal to 2).

\section{Patient characteristics}

In this study, 342 subjects were considered. The number of participants varied between the articles included from 10 to 100, with an average of 29 subjects per study. Amongst the population studied, 53.5\% (183

Table 2: The Jadad Scale.

\section{Question}

(1) Was the study described as randomized?

(2) Was the double blinded correct?

No $=0$

(3) Losses and exclusions were described? Yes $=1$

\begin{tabular}{|l|l|}
\hline (4) The randomization was adequate? & Yes $=1$ \\
\hline & No $=-1$ \\
\hline $\begin{array}{l}\text { (5) The blinding of methodology was } \\
\text { correct? }\end{array}$ & Yes $=1$ \\
\hline
\end{tabular}

volunteers) were men and $46.5 \%$ (159 volunteers) were women. Of these 342 subjects, $45.3 \%$ of them (155 subjects) were included as healthy subjects, whereas $52.6 \%$ of them (187 patients) were included with a specific pathology. In the total number of patients included as healthy, it is important to note that $27.1 \%$ of them (42 subjects) were recruited with an ankle dorsiflexion limitation. Within the different pathologies studied, we can find plantar heel pain (53.5\%), plantar fasciitis $(21.4 \%)$, knee osteoarthritis (13.4\%), nocturnal calf cramps (6.4\%), and calf pain (5.3\%).

\section{MTrPs characteristics}

As an inclusion criterion, the protocol of the different studies established the confirmed presence of latent MTrPs for $29.2 \%$ of participants (100 subjects), active MTrPs for $5.0 \%$ (17 subjects), both active or latent MTrPs for $42.4 \%$ of subjects (145 subjects), and unspecified MTrPs for $21.3 \%$ of volunteers (73 subjects).

\section{Variables}

The following variables were used to measure the different outcomes in the studies (Table 4):

Pain: Evaluated by the use of Visual Analogic Scale (VAS), Pressure Pain Threshold (PPT), or various questionnaires (FHSQ-pain, NRS, LEFS, BPI, SF-MPQ, SF-12).

Jump performance: This variable was measured in one study using the application called "My Jump", which analyzes different jump parameters (jump height, power output, optimal force, and velocity). In another study, jump performance was assessed through the usage of calk marks on the wall.

Table 3: Scientific evidence table.

\begin{tabular}{|c|c|c|c|c|}
\hline Author & Year & Journal & Sample & Evidence level \\
\hline Devereux, et al. [21] & 2018 & Journal of strength and conditioning research & 40 & $\mathrm{I} / 0$ \\
\hline Bandy, et al. [20] & 2017 & International journal of sports physical therapy & 35 & $1 / 4$ \\
\hline Ye, et al. [22] & 2015 & Pain Medicine & 100 & $1 / 5$ \\
\hline Kim, et al. [16] & 2015 & Journal of the American Board of Family Medicine: JABFM & 12 & II - 2/0 \\
\hline Moghtaderi, et al. [26] & 2013 & Advanced biomedical research & 40 & $1 / 2$ \\
\hline Grieve, et al. [2] & 2013 & Manual Therapy & 10 & I/1 \\
\hline Grieve, et al. [25] & 2012 & Journal of bodywork and movement therapies. & 22 & li-3/- \\
\hline Henry, et al. [17] & 2012 & Pain research \& management & 25 & II-2/0 \\
\hline Grieve, et al. [24] & 2009 & Journal of bodywork and movement therapies. & 20 & $1 / 1$ \\
\hline Ge, et al. [23] & 2009 & $\begin{array}{l}\text { Acupuncture in medicine: journal of the British Medical } \\
\text { Acupuncture Society }\end{array}$ & 13 & $1 / 2$ \\
\hline Li, et al. [18] & 2009 & The Clinical journal of pain & 11 & ||$-3 /-$ \\
\hline Ge, et al. [19] & 2008 & Experimental brain research & 14 & I/1 \\
\hline
\end{tabular}


Table 4: Results table: treatment modalities and related outcomes.

\section{Author}

Devereux, et Control group: no intervention

al. [21] Group 1: Dry needling of MTrPs Rectus femoris

Group 3: Dry needling of MTrPs in both
Group 2: Dry needling of MTrPs Medial gastrocnemius

Variables

\section{Results}

Global improvement in all experimental groups and

Jump Performance results are statistically significant when the medial gastrocnemius is treated.

Significant improvement

Bandy, et al. Control group: Sham group (needles not introduced)

[20] Experimental: DN of 4 sites on bilateral gastrocnemius muscle Jump Performance
Ye, et al. [22] Control group: Sham group. Same methods without application of Pulsed radiofrequency.

Experimental: ultrasound-guided pulsed radiofrequency in the gastrocnemius Pulsed radiofrequency treatment once per week. PRF at $42{ }^{\circ} \mathrm{C}$ for 5 minutes and $3 \mathrm{~mL}$ of $0.5 \%$ levobupivacaine was injected into the trigger points.
Kim, et al. Control group: no treatment

[16] Experimental: Injection of $1-2 \mathrm{~mL}$ of $0.25 \%$ lidocaine into each trigger points in the gastrocnemius muscles at 1, 2 and 4 weeks after the first visit. in jump performance with treatment.

Pain reduced and an improvement to the quality
Pain and quality of life of life in the EG. Both results were statistically significant.
Moghtaderi, Control group: ESWT for heel region (3000 shock waves/ et al. [26] session of $0.2 \mathrm{~mJ} / \mathrm{mm}^{2}$ )

Experimental: ESWT for heel region + gastrocnemius trigger points (3000 shock waves/session of $0.2 \mathrm{~mJ} / \mathrm{mm}^{2}$ for the heel region and 400 shock waves/session of $0.2 \mathrm{~mJ} / \mathrm{mm}^{2}$ per each trigger point) \begin{tabular}{l|l} 
Both variables present \\
statistically significant
\end{tabular}

Pain and Insomnia

\section{Grieve, et Control group: no}

al. [2]
Experimental: MTrPs intervention (pressure release) + self MTrP release + home stretching program.
Pain, ROM and

MTrPs prevalence
Both control and experimental groups present a statistically significant pain reduction with improved results in the EG.
Treatment received by the EG allows an inactivation of the ATrPs and an elimination of the LTrPs. An increased ROM dorsiflexion is also observed.

Treatment of MTrPs of both soleus and gastrocnemius muscles allows for statistically significant results in increased ROM.
Grieve, et al. Control group: Sham therapy

[25] Experimental group: 10 min of TrP pressure release treatment to the identified MTrPs in the Gastrocnemius and Soleus ROM followed by $1 \times 10$ s passive stretch.

Henry, et al. Control group: No

[17] Experimental: injections of local anesthetic $(0.25 \%$ bupivacaine, 25-gauge, 1.5-inch needle).
Pain, Mobility and

Depression/Anxiety Levels
EG presents a significant pain reduction and an improvement of mobility. However, no effect on depression or anxiety levels were found.

\section{Treatment of MTrPs} allows for a statistically significant increase of ROM dorsiflexion.

Treatment of MTrPs of Reflex responses of the tibial nerve improvement of the reflex response parameters. 
Li, et al. [18] Control group: EMG-guided intramuscular injection (bolus of either hypertonic saline $(6 \%, 0.1 \mathrm{~mL}$, each), glutamate $(0.1$ $\mathrm{mL}, 0.5 \mathrm{M}$, each), or isotonic saline $(0.9 \%, 0.1 \mathrm{~mL}$, each) in no-MTrPs

Experimental: EMG-guided intramuscular injection (bolus of either hypertonic saline $(6 \%, 0.1 \mathrm{~mL}$, each), glutamate $(0.1$ $\mathrm{mL}, 0.5 \mathrm{M}$, each), or isotonic saline $(0.9 \%, 0.1 \mathrm{~mL}$, each) in Latent MTrPs gastrocnemius.

Injections of glutamate or isotonic saline solution induce an increased level of pain that is statistically significant. This occurs regardless of if the injections are in the non-MTrPs, suggesting a non-nociceptive hypersensitivity at latent MTrPs.

The results showed that glutamate and isotonic saline injections into the latent MTrPs induced higher peak pain intensity than into the non-MTrPs.
Quality of life: The evaluation of this variable was done through the use of the SF-36 questionnaire.

Insomnia: It was assessed using the ISI questionnaire.

Depression or anxiety: These variables were measured through the CES-D and the STAI questionnaires.

Range of motion (ROM) ankle dorsiflexion: The evaluation of this variable was assessed in 3 different studies, in all of them, a classical two-armed plastic goniometer was used.

Mobility: Basic mobility skills (for example, standing from a sitting position on standard arm chair, walking 3 meters, turning, walking back to the chair, and sitting down) were evaluated by the TUG questionnaire.

Reflex responses: The H-reflex latency and the conduction velocity were studied using intramuscular stimulation at MTrPs and non-MTrPs.

\section{Treatment and clinical results}

The treatments used were: injections $(27.0 \%)$, dry needling $(20.9 \%)$, pulsed radiofrequency $(20.4 \%)$, pressure release (17.4\%), ESWT (8.7\%), and intramuscular electrical stimulation (5.7\%) (Table 4).

Injections: This treatment obtains significant results in reducing pain [16-19], in improving the quality of sleep [16], and in improving mobility [17]. However, no improvements were shown for depression or anxiety [17].

Dry needling: This treatment allows significant results in improving jump capacity $[20,21]$.

Electrostimulation: This intervention, obtains significant results regardless of its various methods of execution. In one study, the use of pulsed radiofrequency decreased pain and improved quality of life [22]. In another study, the use of intramuscular electrical stimulation permitted the modification of reflex responses [23].

Ischemic compression: This therapy was used in two studies. One of the studies that used this therapy had seen significant results in increasing ROM of dorsiflexion
$[2,24]$. Whereas the other study had seen non-significant improvement [25]. In one of them, significant results were also obtained in increasing the pressure pain threshold [25].

Extracorporeal shockwave therapy (ESWT): this intervention proved to be significantly efficient in reducing pain [26].

\section{Discussion}

Once the bibliography had been reviewed, regardless of the applied treatment, we observed that intervention on trigger-points (both active or latent) improves the aforementioned symptomatology, with the exception of the patient's state of anxiety and depression [17]. In most of the analyzed studies, when the intervention of TP is compared to the control groups (sham group), the experimental groups improve in jump capacity $[20,21]$ and decrease in pain $[16,17,19,22,26]$. This causes an improved quality of life and athletic ability for the patients as demonstrated in the bibliography regarding other parts of the body.

All the applied treatments can be classified in two groups, invasive and conservative interventions. Some studies that use invasive techniques compare dry needling with no treatment [21] or with placebo needling [20]. Another comparison has been made between the use of electrostimulation in the MTrPs and placebo treatment without performing the electrostimulation [22] or with electrostimulation out of MTrPs area [23]. All of these techniques can be included in the treatments available to a physical therapist in a wide majority of countries. Four studies used injections of substances as interventions [16-19], two of those were compared to a placebo treatment (injection out of the TP area) $[18,19]$ and in the other two there was no control group $[16,17]$. Unlike the first invasive techniques described, those injections cannot be included in the treatments available for a physical therapist.

Of the non-invasive treatments, we observed that the most applied is ischemic compression, which allows pain reduction [2], dorsiflexion improvement [24,25], 
deactivation of the ATrPs and disappearance of the LTrPs [2]. In these studies, the intervention groups in which ischemic compression was applied were compared with control groups in which no intervention was realized. Of all other non-invasive techniques available, the present review has only found one study that applied shock waves [26].

But independently of the intervention, in some articles it was observed that there was a lack of details about the treatment applied $[20,21]$. For example, in the dry needling studies, the technique, doses, and parameters employed were not specified. Additional information such as if the needling was deep or superficial, if it was done using multiple rapid insertions, if the needle was screwed in when inserted, or regarding the number of local twitch responses. Moreover, some aspects of these techniques are still being investigated such as the production of single or multiple local twitch responses [27].

This revision has shown that the methodology in some research is not clear regarding the control and sham group. In the case of the dry needling techniques, the sham strategies were complicated because patients were aware of the treatment that they were or were not receiving. Some studies described how they applicate the tube of the needle without introducing of the needle [20]. Additionally, if you compare the effects of Ischemic compression with a control, it makes it difficult for the researchers to use any blinding strategy $[2,24]$, and the authors choose to not include one at all. In these studies, the researchers have confused the sham group without intervention, the correct strategy should be to apply the same techniques out of the MTrPs. In the case of the injections, the sham strategy is easier because patients feel the same even if they do not know which product is injected.

Myofascial trigger points are one of the most important reasons for musculoskeletal pain and medical consults. The diagnosis of the MTrPs is, however, still in discussion, therefore the clinical symptoms are the principal criteria. For this reason, as suggested in the bibliography, the reclusion of the sample is not always precise. In some cases, the difference between MTrPs that are latent or active is not clear.

Despite the fact that the studies present a good level of evidence according to the US preventive task force, the Jadad scale demonstrates that the methodology could be improved since in many cases neither the random assignment nor the blinding method are explained.

\section{Limitations}

One of the limitations of the present study was the disparity in the quality of the research that was part of this review. Some publications do not specify how the evaluators were masked, or if there was or was not a control group. The percentage of the type of trigger points (active or latent) did not appear in the majority of studies analyzed. Another point that called the attention of the authors, was that there was no study that compared between invasive interventions and noninvasive interventions. Each investigation was realized comparing one treatment technique with same kind of treatment or with a placebo intervention. For the noninvasive method of treatment, like most of the times in physical therapy, the comparison was made with no intervention group due to the difficulty of the application of a good sham treatment.

\section{Future Investigations}

Based on the limitations found in the different studies, more research would be necessary in order to complete the lack of information in the field of physical therapy techniques. For example, it would be necessary to investigate and compare the effects of invasive and non-invasive interventions in the treatment of MTrPs. There should also be more research to confirm the results already found by increasing the sample size or improving the differentiation between LTrPs and ATrPs. A new line of investigation could also be used to analyze the effect of the treatment of MTrPs on skeletal muscle's contractile properties as this area of knowledge is not currently being investigated.

\section{References}

1. Simons DG, Travell JG, Simons LS (1999) Travell \& Simons myofascial pain and dysfunction: upper half of body: Lippincott Williams \& Wilkins.

2. Grieve R, Barnett S, Coghill N, Cramp F (2013) Myofascial trigger point therapy for triceps surae dysfunction: a case series. Man Ther 18: 519-525.

3. Simons DG (1996) Clinical and etiological update of myofascial pain from trigger points. J Musculoskeletal Pain 4: 93-122.

4. McPartland JM, Simons DG (2006) Myofascial trigger points: translating molecular theory into manual therapy. Journal of Manual \& Manipulative Therapy 14: 232-239.

5. Chaitow L (2008) Como conocer, localizar y tratar los puntos gatillo miofasciales. Guía de masaje para terapeutas manuales.

6. Grieve R, Barnett S, Coghill N, Cramp F (2013) The prevalence of latent myofascial trigger points and diagnostic criteria of the triceps surae and upper trapezius: a cross sectional study. Physiotherapy 99: 278-284.

7. Torres-Chica B, Nunez-Samper-Pizarroso C, OrtegaSantiago R, Cleland JA, Salom-Moreno J, et al. (2015) Trigger points and pressure pain hypersensitivity in people with postmeniscectomy pain. Clin J Pain 31: 265-272.

8. Zuil-Escobar JC, Martínez-Cepa CB, Martín-Urrialde JA, Gómez-Conesa A (2016) The prevalence of latent trigger points in lower limb muscles in asymptomatic subjects. PM R 8: 1055-1064.

9. Simons DG (2004) Review of enigmatic MTrPs as a common cause of enigmatic musculoskeletal pain and dysfunction. $J$ Electromyogr Kinesiol 14: 95-107.

10. Aguilera FJ, Martin DP, Masanet RA, Botella AC, Soler LB, 
et al. (2009) Immediate effect of ultrasound and ischemic compression techniques for the treatment of trapezius latent myofascial trigger points in healthy subjects: a randomized controlled study. J Manipulative Physiol Ther 32: 515-520.

11. Ge HY, Arendt-Nielsen L (2011) Latent myofascial trigger points. Curr Pain Headache Rep 15: 386-392.

12. Celik D, Mutlu EK (2013) Clinical implication of latent myofascial trigger point. Curr Pain Headache Rep 17: 353.

13. Jadad AR, Moore RA, Carroll D, Jenkinson C, Reynolds DJM, et al. (1996) Assessing the quality of reports of randomized clinical trials: is blinding necessary? Control Clin Trials 17: 1-12.

14. US Preventive Services Task Force (1989) Guide to clinical preventive services: report of the US Preventive Services Task Force: DIANE publishing.

15. Harris RP, Helfand M, Woolf SH, Lohr KN, Mulrow CD, et al. (2001) Current methods of the US Preventive Services Task Force: a review of the process. Am J Prev Med 20: 21-35.

16. Kim DH, Yoon DM, Yoon KB (2015) The effects of myofascial trigger point injections on nocturnal calf cramps. J Am Board Fam Med 28: 21-27.

17. Henry R, Cahill CM, Wood G, Hroch J, Wilson R, et al. (2012) Myofascial pain in patients waitlisted for total knee arthroplasty. Pain Res Manag 17: 321-327.

18. Li L, Ge H, Yue S, Arendt-Nielsen L (2009) Nociceptive and non-nociceptive hypersensitivity at latent myofascial trigger points. Clin J Pain 25: 132-137.

19. Ge H, Zhang Y, Boudreau S, Yue S, Arendt-Nielsen L (2008) Induction of muscle cramps by nociceptive stimulation of latent myofascial trigger points. Exp Brain Res 187: 623629.
20. Bandy WD, Nelson R, Beamer L (2017) Comparison of Dry Needling Vs. Sham on the Performance of Vertical Jump. Int J Sports Phys Ther 12: 747-751.

21. Devereux F, O’Rourke B, Byrne PJ, Byrne D, Kinsella S (2018) The Effects of Myofascial Trigger Point Release on the Power and Force Production in the Lower Limb Kinetic Chain. J Strength Cond Res 22.

22. Ye L, Mei Q, Li M, Gu M, Ai Z, et al. (2015) A comparative efficacy evaluation of ultrasound-guided pulsed radiofrequency treatment in the gastrocnemius in managing plantar heel pain: a randomized and controlled trial. Pain Med 16: 782-790.

23. Ge HY, Serrao M, Andersen OK, Graven-Nielsen T, ArendtNielsen L (2009) Increased H-reflex response induced by intramuscular electrical stimulation of latent myofascial trigger points. Acupunct Med 27: 150-154.

24. Grieve R, Clark J, Pearson E, Bullock S, Boyer C, et al (2011) The immediate effect of soleus trigger point pressure release on restricted ankle joint dorsiflexion: a pilot randomised controlled trial. J Bodyw Mov Ther 15: 42-49.

25. Grieve R, Cranston A, Henderson A, John R, Malone $G$, et al. (2013) The immediate effect of triceps surae myofascial trigger point therapy on restricted active ankle joint dorsiflexion in recreational runners: A crossover randomised controlled trial. J Bodyw Mov Ther 17: 453-461.

26. Moghtaderi A, Khosrawi S, Dehghan F (2014) Extracorporeal shock wave therapy of gastroc-soleus trigger points in patients with plantar fasciitis: A randomized, placebocontrolled trial. Adv Biomed Res 3: 99.

27. Perreault T, Dunning J, Butts R (2017) The local twitch response during trigger point dry needling: Is it necessary for successful outcomes? J Bodyw Mov Ther 21: 940-947. 